\title{
Research Article \\ Summation Characterization of the Recessive Solution for Half-Linear Difference Equations
}

\author{
Ondřej Došlý ${ }^{1}$ and Simona Fišnarová ${ }^{2}$ \\ ${ }^{1}$ Department of Mathematics and Statistics, Masaryk University, Kotlářská 2, 61137 Brno, Czech Republic \\ ${ }^{2}$ Department of Mathematics, Mendel University of Agriculture and Forestry in Brno, Zemëdělská 1, \\ 61300 Brno, Czech Republic
}

Correspondence should be addressed to Ondřej Došlý, dosly@math.muni.cz

Received 24 June 2009; Accepted 24 August 2009

Recommended by Martin J. Bohner

We show that the recessive solution of the second-order half-linear difference equation $\Delta\left(r_{k} \Phi\left(\Delta x_{k}\right)\right)+c_{k} \Phi\left(x_{k+1}\right)=0, \Phi(x):=|x|^{p-2} x, p>1$, where $r, c$ are real-valued sequences, is closely related to the divergence of the infinite series $\sum^{\infty}\left(r_{k} x_{k} x_{k+1}\left|\Delta x_{k}\right|^{p-2}\right)^{-1}$.

Copyright @ 2009 O. Došlý and S. Fišnarová. This is an open access article distributed under the Creative Commons Attribution License, which permits unrestricted use, distribution, and reproduction in any medium, provided the original work is properly cited.

\section{Introduction}

We consider the second-order half-linear difference equation

$$
\Delta\left(r_{k} \Phi\left(\Delta x_{k}\right)\right)+c_{k} \Phi\left(x_{k+1}\right)=0, \quad \Phi(x):=|x|^{p-2} x, \quad p>1
$$

where $r, c$ are real-valued sequences and $r_{k}>0$, and we investigate properties of its recessive solution.

Qualitative theory of (1.1) was established in the series of the papers of Řehák [1-5] and it is summarized in [6, Chapter 3]. It was shown there that the oscillation theory of (1.1) is very similar to that of the linear equation

$$
\Delta\left(r_{k} \Delta x_{k}\right)+c_{k} x_{k+1}=0
$$

which is the special case $p=2$ in (1.1). We will recall basic facts of the oscillation theory of (1.1) in the following section. 
The concept of the recessive solution of (1.1) has been introduced in [7]. There are several attempts in literature to find a summation characterization of this solution, see [8] and also related references $[9,10]$, which are based on the asymptotic analysis of solutions of (1.1). However, this approach requires the sign restriction of the sequence $c_{k}$ and additional assumptions on the convergence (divergence) of certain infinite series involving sequences $r$ and $c$, see Proposition 2.1 in the following section. Here we use a different approach which is based on estimates for a certain nonlinear function which appears in the Picone-type identity for (1.1).

The recessive solution of (1.1) is a discrete counterpart of the concept of the principal solution of the half-linear differential equation

$$
\left(r(t) \Phi\left(x^{\prime}\right)\right)^{\prime}+c(t) \Phi(x)=0,
$$

which attracted considerable attention in recent years, we refer to the work in [11-15] and the references given therein.

Let us recall the main result of [11] whose discrete version we are going to prove in this paper.

Proposition 1.1. Let $\tilde{x}$ be a solution of (1.3) such that $\tilde{x}^{\prime}(t) \neq 0$ for large $t$.

(i) Let $p \in(1,2]$. If

$$
I(\tilde{x}):=\int^{\infty} \frac{d t}{r(t) \tilde{x}^{2}(t)\left|\tilde{x}^{\prime}(t)\right|^{p-2}}=\infty,
$$

then $\tilde{x}$ is the principal solution of (1.3).

(ii) If $p \geq 2$ and $I(\tilde{x})<\infty$, then $\tilde{x}$ is not the principal solution of (1.3).

The paper is organized as follows. In Section 2 we recall elements of the oscillation theory of (1.1). Section 3 is devoted to technical statements which we use in the proofs of our main results which are presented in Section 4 . Section 5 contains formulation of open problems in our research.

\section{Preliminaries}

Oscillatory properties of (1.1) are defined using the concept of the generalized zero which is defined in the same way as for (1.2), see, for example, [6, Chapter 3],or [16, Chapter 7]. A solution $x$ of (1.1) has a generalized zero in an interval $(m, m+1]$ if $x_{m} \neq 0$ and $x_{m} x_{m+1} r_{m} \leq 0$. Since we suppose that $r_{k}>0$ (oscillation theory of (1.1) generally requires only $r_{k} \neq 0$ ), a generalized zero of $x$ in $(m, m+1]$ is either a "real" zero at $k=m+1$ or the sign change between $m$ and $m+1$. However, (1.1) is said to be disconjugate in a discrete interval $[m, n]$ if the solution $x$ of (1.1) given by the initial condition $x_{m}=0, x_{m+1} \neq 0$ has no generalized zero in $(m, n+1]$. However, (1.1) is said to be nonoscillatory if there exists $m \in \mathbb{N}$ such that is disconjugate on $[m, n]$ for every $n>m$ and is said to be oscillatory in the opposite case. 
If $x$ is a solution of (1.1) such that $x_{k} \neq 0$ in some discrete interval $[m, \infty)$, then $w_{k}=$ $r_{k} \Phi\left(\Delta x_{k} / x_{k}\right)$ is a solution of the associated Riccati type equation

$$
\Delta w_{k}+c_{k}+w_{k}\left(1-\frac{r_{k}}{\Phi\left(\Phi^{-1}\left(r_{k}\right)+\Phi^{-1}\left(w_{k}\right)\right)}\right)=0
$$

where $\Phi^{-1}(x)=|x|^{q-2} x$ is the inverse function of $\Phi$ and $q=p /(p-1)$ is the conjugate number to $p$. Moreover, if $x$ has no generalized zero in $[m, \infty)$, then $\Phi^{-1}\left(r_{k}\right)+\Phi^{-1}\left(w_{k}\right)>0, k \in[m, \infty)$. If we suppose that (1.1) is nonoscillatory, among all solutions of (2.1) there exists the socalled distinguished solution $\widetilde{w}$ which has the property that there exists an interval $[m, \infty)$ such that any other solution $w$ of (2.1) for which $\Phi^{-1}\left(r_{k}\right)+\Phi^{-1}\left(w_{k}\right)>0, k \in[m, \infty)$, satisfies $w_{k}>\widetilde{w}_{k}, k \in[m, \infty)$. Therefore, the distinguished solution of (2.1) is, in a certain sense, minimal solution of this equation near $\infty$, and sometimes it is called the minimal solution of (2.1). If $\tilde{w}$ is the distinguished solution of (2.1), then the associated solution of (1.1) given by the formula

$$
\tilde{x}_{k}=\prod_{j=m}^{k-1}\left[1+\Phi^{-1}\left(\frac{\tilde{w}_{j}}{r_{j}}\right)\right]
$$

is said to be the recessive solution of (1.1), see [7]. Note that in the linear case $p=2$ a solution $\tilde{x}$ of (1.2) is recessive if and only if

$$
\sum \frac{1}{r_{k} \tilde{x}_{k} \tilde{x}_{k+1}}=\infty
$$

At the end of this section, for the sake of comparison, we recall the main results of $[8,17]$, where summation characterizations of recessive solutions of (1.1) are investigated using the asymptotic analysis of the solution space of (1.1).

Proposition 2.1. Let $x$ be a solution of (1.1).

(i) Suppose that $c_{k}<0$, then $x$ is the recessive solution of (1.1) if and only if

$$
\sum^{\infty} \frac{1}{r_{k}^{q-1} x_{k} x_{k+1}}=\infty
$$

(ii) Suppose that $c_{k}>0, \sum^{\infty} r_{k}^{1-q}<\infty$, and

$$
\sum^{\infty} c_{k} \Phi\left(\sum_{j=k+1}^{\infty} r_{j}^{1-q}\right)<\infty
$$


If $x$ is the recessive solution of (1.1), then

$$
\sum^{\infty} \frac{1}{r_{k} x_{k} x_{k+1}\left|\Delta x_{k}\right|^{p-2}}=\infty
$$

(iii) Suppose that $c_{k}>0, \Sigma^{\infty} c_{k}<\infty$, and $\sum^{\infty} r_{k}^{1-q}<\infty$. Then $x$ is the recessive solution if and only if (2.4) holds.

In cases (i) and (iii), the previous proposition gives necessary and sufficient condition for a solution $x$ to be recessive. The reason why under assumptions in (i) or (iii) it is possible to formulate such a condition is that there is a substantial difference in asymptotic behavior of recessive and dominant solutions (i.e., solutions which are linearly independent of the recessive solution). This difference enables to "separate" the recessive solution from dominant ones and to formulate for it a necessary and sufficient condition (2.4). We refer to $[8,17]$ and also to $[9,10]$ for more details.

\section{Technical Results}

Throughout the rest of the paper we suppose that (1.1) is nonoscillatory and $h$ is its solution. Denote

$$
\begin{gathered}
v_{k}^{*}:=r_{k} h_{k}\left(\Phi\left(h_{k}\right)+\Phi\left(\Delta h_{k}\right)\right), \quad R_{k}:=\frac{2}{q} r_{k} h_{k} h_{k+1}\left|\Delta h_{k}\right|^{p-2}, \\
G_{k}:=r_{k} h_{k} \Phi\left(\Delta h_{k}\right),
\end{gathered}
$$

and define the function

$$
H(k, v):=v+r_{k} h_{k+1} \Phi\left(\Delta h_{k}\right)-\frac{r_{k}\left(v+G_{k}\right)\left|h_{k+1}\right|^{p}}{\Phi\left(\left|h_{k}\right|^{q} \Phi^{-1}\left(r_{k}\right)+\Phi^{-1}\left(v+G_{k}\right)\right)} .
$$

Lemma 3.1. Put

$$
v_{k}:=\left|h_{k}\right|^{p}\left(w_{k}-\tilde{w}_{k}\right),
$$

where $\tilde{w}_{k}=r_{k} \Phi\left(\Delta h_{k} / h_{k}\right)$ is a solution of (2.1) and $w_{k}$ is any sequence satisfying $r_{k}+w_{k} \neq 0$. Then the following statements hold:

(i) $w_{k}$ is a solution of (2.1) if and only if $v_{k}$ is a solution of

$$
\Delta v_{k}+H\left(k, v_{k}\right)=0
$$

(ii) $H(k, v) \geq 0$ for $v>-v_{k}^{*}$ with the equality if and only if $v=0$;

(iii) $r_{k}+w_{k}>0$ if and only if $v_{k}+v_{k}^{*}>0$;

(iv) let $v$ be a solution of (3.4) and suppose that $v_{m}<0$ for some $m \in \mathbb{N}$, that is, $w_{m}<\widetilde{w}_{m}$, then $v_{m+1}>0$ if and only if $v_{m}+v_{m}^{*}<0$. 
Proof. The statements (i), (ii) are consequences of [18, Lemma 2.5].

(iii) We have

$$
\begin{aligned}
r_{k}+w_{k} & =r_{k}+\left|h_{k}\right|^{-p} v_{k}+\tilde{w}_{k} \\
& =r_{k}+\left|h_{k}\right|^{-p} v_{k}+r_{k} \Phi\left(\frac{\Delta h_{k}}{h_{k}}\right) \\
& =\left|h_{k}\right|^{-p}\left[v_{k}+r_{k} h_{k}\left(\Phi\left(h_{k}\right)+\Phi\left(\Delta h_{k}\right)\right)\right] \\
& =\left|h_{k}\right|^{-p}\left(v_{k}+v_{k}^{*}\right) .
\end{aligned}
$$

(iv) We have

$$
\begin{aligned}
v_{m+1}= & v_{m}-H\left(m, v_{m}\right) \\
= & r_{m} h_{m+1}\left[\frac{\Phi\left(h_{m+1}\right)\left(v_{m}+G_{m}\right)}{\Phi\left(\left|h_{m}\right|^{q} \Phi^{-1}\left(r_{m}\right)+\Phi^{-1}\left(v_{m}+G_{m}\right)\right)}-\Phi\left(\Delta h_{m}\right)\right] \\
= & r_{m} h_{m+1}\left[\frac{\Phi\left(h_{m+1}\right) w_{m}}{\Phi\left(\Phi^{-1}\left(r_{m}\right)+\Phi^{-1}\left(w_{m}\right)\right)}-\Phi\left(\Delta h_{m}\right)\right] \\
= & r_{m} h_{m+1} \Phi\left(h_{m}\right)\left[\Phi\left(\frac{h_{m+1}}{h_{m}}\right) \frac{w_{m}}{\Phi\left(\Phi^{-1}\left(r_{m}\right)+\Phi^{-1}\left(w_{m}\right)\right)}-\Phi\left(\frac{\Delta h_{m}}{h_{m}}\right)\right] \\
= & \frac{r_{m} h_{m+1} \Phi\left(h_{m}\right)}{\Phi\left(\Phi^{-1}\left(r_{m}\right)+\Phi^{-1}\left(w_{m}\right)\right)} \\
& \times\left[\Phi\left(\frac{h_{m+1} \Phi^{-1}\left(w_{m}\right)}{h_{m}}\right)-\Phi\left(\frac{\Delta h_{m}}{h_{m}}\right) \Phi\left(\Phi^{-1}\left(r_{m}\right)+\Phi^{-1}\left(w_{m}\right)\right)\right] .
\end{aligned}
$$

Denote by $A$ the expression in brackets, then

$$
\begin{aligned}
\operatorname{sgn} A & =\operatorname{sgn}\left[\frac{h_{m+1} \Phi^{-1}\left(w_{m}\right)}{h_{m}}-\left(\frac{h_{m+1}}{h_{m}}-1\right)\left(\Phi^{-1}\left(r_{m}\right)+\Phi^{-1}\left(w_{m}\right)\right)\right] \\
& =\operatorname{sgn}\left[\Phi^{-1}\left(r_{m}\right)+\Phi^{-1}\left(w_{m}\right)-\frac{\left(h_{m}+\Delta h_{m}\right) \Phi^{-1}\left(r_{m}\right)}{h_{m}}\right] \\
& =\operatorname{sgn}\left[\Phi^{-1}\left(w_{m}\right)-\Phi^{-1}\left(\tilde{w}_{m}\right)\right]=\operatorname{sgn} v_{m}=-1 .
\end{aligned}
$$

Consequently,

$$
v_{m+1}>0 \Longleftrightarrow \Phi^{-1}\left(r_{m}\right)+\Phi^{-1}\left(w_{m}\right)<0,
$$

that is, the statement holds according to the statement (iii) of this lemma. 
Lemma 3.2. Let $v^{*}, R, G, H$ be defined by (3.1), (3.2) and suppose that $h_{k} \Delta h_{k}<0$ for large $k$. Then one has the following inequalities for large $k$.

If $p \in(1,2]$, then $v_{k}^{*} \leq R_{k}$ and

$$
v-H(k, v) \leq \frac{R_{k} v}{R_{k}+v} \quad \text { for } v \in\left(-v_{k}^{*}, 0\right]
$$

If $p \geq 2$, then $v_{k}^{*} \geq R_{k}$ and

$$
v-H(k, v) \geq \frac{R_{k} v}{R_{k}+v} \quad \text { for } v \in\left(-R_{k}, 0\right]
$$

Proof. We have (with using the Lagrange mean value theorem)

$$
\begin{aligned}
v_{k}^{*} & =r_{k} h_{k}\left(\Phi\left(h_{k}\right)+\Phi\left(\Delta h_{k}\right)\right) \\
& =r_{k} h_{k} \Phi\left(h_{k+1}\right)\left[\Phi\left(\frac{h_{k}}{h_{k+1}}\right)-\Phi\left(-\frac{\Delta h_{k}}{h_{k+1}}\right)\right] \\
& =r_{k} h_{k} \Phi\left(h_{k+1}\right) \Phi^{\prime}(\xi),
\end{aligned}
$$

where $-\Delta h_{k} / h_{k+1} \leq \xi \leq h_{k} / h_{k+1}$ and hence $\xi \geq\left|\Delta h_{k} / h_{k+1}\right|$.

Thus, if $p \in(1,2]$,

$$
\begin{aligned}
v_{k}^{*} & =(p-1) r_{k} h_{k} \Phi\left(h_{k+1}\right)|\xi|^{p-2} \leq(p-1) r_{k} h_{k} \Phi\left(h_{k+1}\right)\left|\frac{\Delta h_{k}}{h_{k+1}}\right|^{p-2} \\
& =\frac{1}{q-1} r_{k} h_{k} h_{k+1}\left|\Delta h_{k}\right|^{p-2} \leq R_{k}
\end{aligned}
$$

and in the case $p \geq 2$, we obtain

$$
v_{k}^{*} \geq R_{k} .
$$

Next we proceed similarly as in [18, Lemma 2.6]. Inequalities (3.9), (3.10) can be written in the equivalent forms:

$$
\begin{gathered}
\left(R_{k}+v\right) H(k, v) \geq v^{2}, \quad v \in\left(-v_{k}^{*}, 0\right] \text { for } p \in(1,2], \\
\left(R_{k}+v\right) H(k, v) \leq v^{2}, \quad v \in\left(-R_{k}, 0\right] \text { for } p \geq 2 .
\end{gathered}
$$


Denote $F(k, v):=\left(R_{k}+v\right) H(k, v)-v^{2}$ and let $v>-v_{k}^{*}$. Then

$$
\begin{aligned}
H_{v}(k, v) & =1-\frac{r_{k}^{q}\left|h_{k}\right|^{q}\left|h_{k+1}\right|^{p}}{\left(\left|h_{k}\right|^{q} \Phi^{-1}\left(r_{k}\right)+\Phi^{-1}\left(v+G_{k}\right)\right)^{p}} \\
H_{v v}(k, v) & =\frac{q r_{k}^{q}\left|h_{k}\right|^{q}\left|h_{k+1}\right|^{p}\left|v+G_{k}\right|^{q-2}}{\left(\left|h_{k}\right|^{q} \Phi^{-1}\left(r_{k}\right)+\Phi^{-1}\left(v+G_{k}\right)\right)^{p+1}} \\
H_{v v v}(k, 0) & =\frac{q}{r_{k}^{2} h_{k}^{2} h_{k+1}^{2}\left(\Delta h_{k}\right)^{2 p-3}}\left[(q-2) h_{k+1}-(2 q-1) \Delta h_{k}\right]
\end{aligned}
$$

Consequently, $F(k, 0)=F_{v}(k, 0)=F_{v v}(k, 0)=0$ and

$$
\begin{aligned}
F_{v v v}(k, 0) & =R_{k} H_{v v v}(k, 0)+3 H_{v v}(k, 0) \\
& =\frac{2}{r_{k} h_{k} h_{k+1} \Phi\left(\Delta h_{k}\right)}\left[(q-2) h_{k+1}-(2 q-1) \Delta h_{k}\right]+\frac{3 q}{r_{k} h_{k} h_{k+1}\left|\Delta h_{k}\right|^{p-2}} \\
& =\frac{1}{r_{k} h_{k} h_{k+1} \Phi\left(\Delta h_{k}\right)}\left[2(q-2)\left(h_{k}+\Delta h_{k}\right)+(2-q) \Delta h_{k}\right] \\
& =\frac{q-2}{r_{k} h_{k} h_{k+1} \Phi\left(\Delta h_{k}\right)}\left[h_{k}+h_{k}+\Delta h_{k}\right] \\
& =\frac{q-2}{r_{k} h_{k} h_{k+1} \Phi\left(\Delta h_{k}\right)}\left[h_{k}+h_{k+1}\right] .
\end{aligned}
$$

Hence, in view of the assumption $h_{k} \Delta h_{k}<0, \operatorname{sgn} F_{v v v}(k, 0)=-\operatorname{sgn}(q-2)$. It follows that

$$
\operatorname{sgn} F(k, v)=\operatorname{sgn} F_{v v}(k, v)=\operatorname{sgn}(q-2)
$$

in some left neighborhood of $v=0$, and the function $F$ is positive, decreasing, and convex for $p \in(1,2]$, and is negative, increasing, and concave for $p>2$ (with respect to $v$ ). Hence, both the inequalities (3.14) and (3.15) are satisfied in some left neighborhood of $v=0$. The proof will be completed by showing that $F_{v v}(k, v)$ has constant sign on the given intervals. By a direct computation,

$$
\begin{aligned}
F_{v v}(k, v) & =2 H_{v}(k, v)+\left(R_{k}+v\right) H_{v v}(k, v)-2 \\
& =-\frac{2 r_{k}^{q}\left|h_{k}\right|^{q}\left|h_{k+1}\right|^{p}}{\left(\left|h_{k}\right|^{q} \Phi^{-1}\left(r_{k}\right)+\Phi^{-1}\left(v+G_{k}\right)\right)^{p}}+\frac{q r_{k}^{q}\left|h_{k}\right|^{q}\left|h_{k+1}\right|^{p}\left|v+G_{k}\right|^{q-2}\left(R_{k}+v\right)}{\left(\left|h_{k}\right|^{q} \Phi^{-1}\left(r_{k}\right)+\Phi^{-1}\left(v+G_{k}\right)\right)^{p+1}} \\
& =\frac{r_{k}^{q}\left|h_{k}\right|^{q}\left|h_{k+1}\right|^{p}}{\left(\left|h_{k}\right|^{q} \Phi^{-1}\left(r_{k}\right)+\Phi^{-1}\left(v+G_{k}\right)\right)^{p+1}} A(k, v),
\end{aligned}
$$


where

$$
\begin{aligned}
A(k, v) & :=-2\left|h_{k}\right|^{q} \Phi^{-1}\left(r_{k}\right)-2 \Phi^{-1}\left(v+G_{k}\right)+q\left|v+G_{k}\right|^{q-2}\left(R_{k}+v\right) \\
& =(q-2) \Phi^{-1}\left(v+G_{k}\right)+q\left(R_{k}-G_{k}\right)\left|v+G_{k}\right|^{q-2}-2\left|h_{k}\right|^{q} \Phi^{-1}\left(r_{k}\right) .
\end{aligned}
$$

Hence

$$
\operatorname{sgn} A(k, v)=\operatorname{sgn} F_{v v}(k, v) \text { for } v>-v_{k^{\prime}}^{*}
$$

and from (3.18)

$$
\operatorname{sgn} A(k, v)=\operatorname{sgn}(q-2)
$$

in some left neighborhood of $v=0$.

Moreover, for $v<0$

$$
\begin{aligned}
A_{v}(k, v) & =(q-2) \operatorname{sgn}\left(v+G_{k}\right)\left|v+G_{k}\right|^{q-3}\left[(q-1)\left(v+G_{k}\right)+q\left(R_{k}-G_{k}\right)\right] \\
& =-(q-2)\left|v+G_{k}\right|^{q-3}\left[(q-1) v-G_{k}+q R_{k}\right],
\end{aligned}
$$

and $A_{v}(k, v)=0$ (for $v<0$ ) if and only if

$$
v=\widetilde{v}_{k}:=\frac{1}{q-1}\left(G_{k}-q R_{k}\right)=-\frac{1}{q-1} r_{k} h_{k}\left|\Delta h_{k}\right|^{p-2}\left(h_{k}+h_{k+1}\right) .
$$

Next we distinguish between the cases $p \in(1,2]$ and $p \geq 2$.

If $p \in(1,2]$, then using (3.12),

$$
\tilde{v}_{k} \leq-\frac{1}{q-1} r_{k} h_{k} h_{k+1}\left|\Delta h_{k}\right|^{p-2} \leq-v_{k^{\prime}}^{*}
$$

hence $A(k, v)$ is decreasing on $\left(-v_{k}^{*}, 0\right)$ and in view of (3.22) it means that $A(k, v)$ and consequently from (3.21) also $F_{v v}(k, v)$ is positive for $v \in\left(-v_{k}^{*}, 0\right)$. Hence, (3.14) holds.

Similarly, if $p \geq 2$, then

$$
\tilde{v}_{k} \leq-\frac{1}{q-1} r_{k} h_{k} h_{k+1}\left|\Delta h_{k}\right|^{p-2} \leq-R_{k}
$$

hence $A(k, v)$ is increasing for $v \in\left(-R_{k}, 0\right)$ and from (3.22) we have that $A(k, v)$ and hence also $F_{v v}(k, v)$ is negative for $v \in\left(-R_{k}, 0\right)$. This means that (3.15) is satisfied. 


\section{Main Results}

Theorem 4.1. Suppose $p \in(1,2]$ and let $h$ be a solution of (1.1) such that $h_{k} \Delta h_{k}<0$ for large $k$. If

$$
\sum^{\infty} \frac{1}{r_{k} h_{k} h_{k+1}\left|\Delta h_{k}\right|^{p-2}}=\infty
$$

then $h$ is the recessive solution.

Proof. Denote by $\widetilde{w}_{k}=r_{k} \Phi\left(\Delta h_{k} / h_{k}\right)$ the associated solution of (2.1) and let $w_{k}$ be a solution of (2.1) generated by another solution (linearly independent of $h$ ) of (1.1). Then, it follows from Lemma 3.1 that $v_{k}=\left|h_{k}\right|^{p}\left(w_{k}-\widetilde{w}_{k}\right)$ is a solution of (3.4), that is,

$$
v_{k+1}=v_{k}-H\left(k, v_{k}\right),
$$

and suppose that this solution satisfies the condition $v_{N}<0$. This means that $w_{N}<\widetilde{w}_{N}$ and to prove that $h$ is the recessive solution of (1.1), we need to show that there exists $m \geq N$ such that $r_{m}+w_{m} \leq 0$, that is, according to Lemma 3.1, $v_{m}+v_{m}^{*} \leq 0$. Suppose by contradiction that $v_{k}+v_{k}^{*}>0$ for $k \geq N$. According to Lemma 3.1 (iv), it means that $v_{k}<0$ for $k \geq N$, that is, $v_{k} \in\left(-v_{k}^{*}, 0\right)$. Then we have from Lemma 3.2 that $v_{k}+R_{k}>0$ and

$$
v_{k+1} \leq \frac{R_{k} v_{k}}{R_{k}+v_{k}} \quad \text { for } k \geq N
$$

Next, consider the equation

$$
u_{k+1}=\frac{R_{k} u_{k}}{R_{k}+u_{k}}
$$

and let $u_{k}$ be its solution satisfying $u_{N}=v_{N}$. However, (4.4) is equivalent to

$$
-\Delta u_{k}=\frac{u_{k}^{2}}{R_{k}+u_{k}}
$$

that is,

$$
-\frac{\Delta u_{k}}{u_{k} u_{k+1}}=\frac{u_{k}}{u_{k+1}\left(R_{k}+u_{k}\right)}=\frac{1}{R_{k}}
$$

where we have substituted for $u_{k+1}$ from (4.4) in the denominator. Hence

$$
\frac{1}{u_{k+1}}=\frac{1}{u_{k}}+\frac{1}{R_{k}}
$$


and we obtain

$$
u_{k}=\frac{1}{1 / u_{N}+\sum_{j=N}^{k-1}\left(1 / R_{j}\right)}
$$

Condition (4.1) implies that there exists $m \geq N$ such that $u_{m}<0$ and either $u_{m+1}>0$ or $u_{m+1}$ is not defined. This means that $R_{m}+u_{m} \leq 0$ (from (4.4)). On the other hand, (4.3) together with (4.4) and the fact that $R_{k} x /\left(R_{k}+x\right)$ is increasing with respect to $x$ on $\left(-v_{k}^{*}, 0\right)$ imply that $v_{k} \leq u_{k}$ for $k \geq N$. Since $v_{k}+R_{k}>0$ for $k \geq N$, we have $u_{k}+R_{k}>0$ for $k \geq N$, a contradiction.

Theorem 4.2. Suppose $p \geq 2$ and let $h$ be a solution of (1.1) such that $h_{k} \Delta h_{k}<0$ for large $k$. If

$$
\sum^{\infty} \frac{1}{r_{k} h_{k} h_{k+1}\left|\Delta h_{k}\right|^{p-2}}<\infty
$$

then $h$ is not the recessive solution.

Proof. Similarly, as in the proof of Theorem 4.1, denote $\widetilde{w}_{k}=r_{k} \Phi\left(\Delta h_{k} / h_{k}\right)$ and let $w_{k}$ be a solution of (2.1) generated by another solution (linearly independent of $h$ ) of (1.1). Then $v_{k}=\left|h_{k}\right|^{p}\left(w_{k}-\widetilde{w}_{k}\right)$ is a solution of (3.4), that is,

$$
v_{k+1}=v_{k}-H\left(k, v_{k}\right),
$$

and suppose that this solution satisfies the condition $v_{N}<0,\left|v_{N}\right|$ being sufficiently small (will be specified later). Hence $w_{N}<\tilde{w}_{N}$ and we have to show that $r_{k}+w_{k}>0$ for $k \geq N$, that is, $v_{k}+v_{k}^{*}>0$ for $k \geq N$.

Let $u_{k}$ be a solution of (4.4) and suppose that $u_{N}=v_{N}$. Hence, similarly as in the proof of Theorem 4.1, we obtain

$$
u_{k}=\frac{1}{1 / u_{N}+\sum_{j=N}^{k-1}\left(1 / R_{j}\right)} .
$$

If $\left|u_{N}\right|$ is sufficiently small, then condition (4.9) implies that $u_{k}<0$ for $k \geq N$ and from (4.4), we have $R_{k}+u_{k}>0$ for $k \geq N$. Consequently, from Lemma 3.2 we obtain that $v_{k}^{*} \geq R_{k}$ and

$$
u_{k}-H\left(k, u_{k}\right) \geq \frac{R_{k} u_{k}}{R_{k}+u_{k}}=u_{k+1} \quad \text { for } k \geq N \text {. }
$$

Moreover, since $x-H(k, x)$ is increasing with respect to $x$ on $\left(-R_{k}, 0\right)$, we obtain from (4.12) that $v_{k} \geq u_{k}$ for $k \geq N$. Hence $R_{k}+v_{k}>0$ for $k \geq N$ and hence also $v_{k}^{*}+v_{k}>0$ for $k \geq N$.

\section{Applications and Open Problems}

(i)Theorems 4.1 and 4.2, as formulated in the previous section, apply only to positive decreasing (or negative increasing) solutions of (1.1). The reason is that we have been able to 
prove inequalities (3.9), (3.10) only when $G=r h \Phi(\Delta h)<0$. We conjecture that Theorems 4.1 and 4.2 remain to hold for every solution of (1.1) for which $\Delta h_{k} \neq 0$ for large $k$. To justify this conjecture, consider the function

$$
\mathcal{f}_{k}(v)=H(k, v) / v(v-H(k, v)) .
$$

By an easy computation one can find that inequalities (3.9), (3.10) are equivalent to the inequalities

$$
\mathcal{F}_{k}(v) \geq \frac{1}{R_{k}}, \quad p \in(1,2], \quad \mathcal{F}_{k}(v) \leq \frac{1}{R_{k}}, \quad p \in[2, \infty) .
$$

However, if $G_{k}>0$, that is, $-G_{k}<0$, we have

$$
\mp_{k}\left(-G_{k}\right)=\frac{1}{r_{k} h_{k} h_{k+1}\left|\Delta h_{k}\right|^{p-2}}=\frac{2}{q R_{k}}
$$

so inequalities (3.9), (3.10) are no longer valid in this case. Numerical computations together with a closer examination of the graph of the function $\mathcal{F}$ lead to the following conjecture.

Conjecture 5.1. Let $h_{k}, h_{k+1}>0, \Delta h_{k} \neq 0$, and $R_{k}^{*}:=(q-1) r_{k} h_{k} h_{k+1}\left|\Delta h_{k}\right|^{p-2}$. Then for $v \in$ $\left(-v_{k}^{*}, \infty\right)$ one has

$$
\mathscr{F}_{k}(v) \geq \frac{1}{R_{k}^{*}} \quad \text { for } p \in(1,2], \quad \mathcal{F}_{k}(v) \leq \frac{1}{R_{k}^{*}} \quad \text { for } p \in[2, \infty) .
$$

To explain this conjecture in more details, consider the case $p \in(1,2]$, the case $p \geq 2$ can be treated analogically. We have (we skip the index $k$, only indices different from $k$ are written explicitly)

$$
\begin{aligned}
\mathcal{F}(\infty) & :=\lim _{v \rightarrow \infty} \mathcal{f}(v)=\frac{1}{r h_{k+1}\left[\Phi\left(h_{k+1}\right)-\Phi(\Delta h)\right]} \\
& =\frac{1}{r \Phi(h) h_{k+1}\left[\Phi\left(h_{k+1} / h\right)-\Phi(\Delta h / h)\right]} \\
& =\frac{(q-1)|\xi|^{2-p}}{r \Phi(h) h_{k+1}}
\end{aligned}
$$

where $\Delta h / h \leq \xi \leq h_{k+1} / h$. If $\Delta h>0$, the direct substitution yields

$$
\mathcal{F}(\infty) \geq \frac{(q-1)}{r h h_{k+1}|\Delta h|^{p-2}} \geq \frac{1}{(q-1) r h h_{k+1}|\Delta h|^{p-2}}=\frac{1}{R^{*}} .
$$


If $\Delta h<0$, then $|\Delta h|<h$ and we proceed as follows. For $p \in(1,2]$, the function $\Phi$ is concave for nonnegative arguments, so for $x, y \geq 0$, we have the inequality

$$
\Phi\left(\frac{x+y}{2}\right) \geq \frac{1}{2}[\Phi(x)+\Phi(y)]
$$

We substitute $x=h_{k+1} / h, y=-\Delta h / h$, then $x+y=1$, that is, $2^{2-p} \geq \Phi(x)+\Phi(y)$. Hence we have

$$
\begin{aligned}
\mathcal{F}(\infty) & =\frac{1}{r h_{k+1} \Phi(h)\left[\Phi\left(h_{k+1} / h\right)-\Phi(\Delta h / h)\right]} \\
& \geq \frac{1}{2^{2-p} r h_{k+1} \Phi(h)}=\frac{|h|^{2-p}}{2^{2-p} r h_{k+1} h} .
\end{aligned}
$$

Hence

$$
\mathcal{F}(\infty) \geq \frac{|h|^{2-p}}{2^{2-p} r h_{k+1} h} \geq \frac{|\Delta h|^{2-p}}{2^{2-p} r h_{k+1} h}
$$

Next we prove that $(q-1) \geq 2^{2-p}$ for $p \in(1,2]$. Denote $t=q-1=1 /(p-1)$, then we need to prove the inequality $g(t):=t-2 \cdot 2^{-1 / t} \geq 0$ for $t \in[1, \infty)$. A standard investigation of the graph of the function $t \rightarrow 2 \cdot 2^{-1 / t}$ shows that the required inequality really holds, so we have

$$
\mathcal{F}(\infty) \geq \frac{1}{(q-1) r h_{k+1} h|\Delta h|^{p-2}}=\frac{1}{R^{*}} .
$$

By a similar computation we find that

$$
\begin{gathered}
\mathcal{F}(0)=\lim _{v \rightarrow 0} \mathcal{F}(v)=\frac{1}{R} \leq \frac{1}{R^{*}}, \quad v^{*} \leq R^{*}, \\
\mathcal{F}\left(-v^{*}+\right)=\lim _{v \rightarrow-v^{*}+} \mathcal{F}(v)=\frac{1}{v^{*}} \geq \frac{1}{R_{k}^{*}}, \quad \mathcal{F}^{\prime}\left(-v^{*}+\right)<0, \\
\mathcal{F}^{\prime}(-G)<0 \quad \text { if } G<0, \quad \mathcal{F}^{\prime}(-G)>0 \quad \text { if } G>0, \\
\mathcal{F}^{\prime}(0)<0 \quad \text { if } G<0, \quad \mathcal{F}^{\prime}(0)>0 \quad \text { if } G>0 .
\end{gathered}
$$

These computations lead to the conjecture that $\mathcal{F}$ attains its global minimum at a point in $\left(-v^{*},-G\right)$ if $G>0$ and at a point in $(-G, \infty)$ if $G<0$. Numerical computations suggest that this minimum is $1 /\left(c r h h_{k+1}|\Delta h|^{p-2}\right)$, where $1 \leq c \leq q-1$.

Having proved inequalities (5.4), Theorems 4.1 and 4.2 could be proved for any positive $h$ with $\Delta h \neq 0$ in the same way as in the previous section, it is only sufficient to replace $R=(2 / q) r h h_{k+1}|\Delta h|^{p-2}$ by $R^{*}=(q-1) r h h_{k+1}|\Delta h|^{p-2}$. 
(ii) A typical example of (1.1) to which Theorems 4.1 and 4.2 apply is (1.1) with

$$
\sum^{\infty} r_{k}^{1-q}<\infty, \quad c_{k}>0, \quad \sum^{\infty} c_{k}=\infty
$$

since under these assumption all positive solutions of (1.1) are decreasing, see [19]. However, one can apply indirectly Theorems 4.1 and 4.2 also to (1.1) with

$$
\sum^{\infty} r_{k}^{1-q}=\infty, \quad c_{k}>0
$$

(and $\sum^{\infty} c_{k}<\infty$, otherwise (1.1) would be oscillatory, see [16, Theorem 8.2.14] ), even if all positive solutions of (1.1) are increasing in this case. The method which enables to overcome this difficulty is the so-called reciprocity principle, which can be explained as follows.

Suppose that $c_{k} \neq 0$ in (1.1) and let $u_{k}:=r_{k} \Phi\left(\Delta x_{k}\right)$. Then by a direct computation one can verify that $u$ solves the so-called reciprocal equation:

$$
\Delta\left(\frac{1}{\Phi^{-1}\left(c_{k}\right)} \Phi^{-1}\left(\Delta u_{k}\right)\right)+r_{k+1}^{1-q} \Phi^{-1}\left(u_{k+1}\right)=0
$$

Moreover, if $c_{k}$ does not change its sign for large $k,(1.1)$ is nonoscillatory if and only if (5.14) is nonoscillatory, see [9]. The following statement relates recessive solutions of (1.1) and (5.14). A similar statement can be found in [9], but our proof differs from that given in [9].

Theorem 5.2. Suppose that (1.1) is nonoscillatory and (5.12) or (5.13) holds. If a solution $h$ of (1.1) is recessive, then $\tilde{u}:=r \Phi(\Delta h)$ is the recessive solution of (5.14).

Proof. First suppose that (5.13) holds and let $\widetilde{w}=r \Phi(\Delta h / h)$ be the distinguished solution of (2.1). Assumption (5.13) implies that $\tilde{w}_{k}>0$ for large $k$, see [7]. The solution $v$ of the Riccati equation

$$
v_{k+1}+r_{k+1}^{1-q}-\frac{c_{k}^{1-q} v_{k}}{\Phi^{-1}\left(c_{k}^{-1}+\Phi\left(v_{k}\right)\right)}=0
$$

associated with (5.14) is given by $v=\left(c^{1-q} \Phi^{-1}(\Delta u)\right) / \Phi^{-1}(u)$ and we have the following relationship between solutions of (5.15) and (2.1) (no index means again the index $k$ ):

$$
\begin{aligned}
v & =\frac{c^{1-q} \Phi^{-1}(\Delta u)}{\Phi^{-1}(u)}=\frac{c^{1-q} \Phi^{-1}\left(-c \Phi\left(x_{k+1}\right)\right)}{\Phi^{-1}(r \Phi(\Delta x))}=-\frac{x_{k+1}}{\Phi^{-1}(r) \Delta x}=-\frac{x+\Delta x}{\Phi^{-1}(r) \Delta x} \\
& =-\frac{1+\Delta x / x}{\Phi^{-1}(r)(\Delta x / x)}=-\frac{1+\Phi^{-1}(w) / \Phi^{-1}(r)}{\Phi^{-1}(w)}=-\frac{\Phi^{-1}(r)+\Phi^{-1}(w)}{\Phi^{-1}(r) \Phi^{-1}(w)} .
\end{aligned}
$$


Since the function

$$
x \rightarrow-\frac{\Phi^{-1}(r)+\Phi^{-1}(x)}{\Phi^{-1}(r) \Phi^{-1}(x)}
$$

is increasing for $x \in \mathbb{R} \backslash\{0\}$, the inequality $0<\widetilde{w}_{k}<w_{k}$ for large $k$ and for any solution $w \neq \tilde{w}$ of (2.1) implies the inequality $0>v_{k}>\widetilde{v}_{k}$, where

$$
\widetilde{v}=\frac{c^{1-q} \Phi^{-1}\left(\Delta \tilde{u}_{k}\right)}{\Phi^{-1}\left(\widetilde{u}_{k}\right)}=-\frac{\Phi^{-1}(r)+\Phi^{-1}(\tilde{w})}{\Phi^{-1}(r) \Phi^{-1}(\widetilde{w})}
$$

and $v$ is any other solution of (5.15). Consequently, $\widetilde{v}$ is the distinguished solution of (5.15) and hence $\tilde{u}$ is the recessive solution of (5.14).

Now suppose that (5.12) holds. Then all solutions $w$ of (2.1) satisfying $r_{k}+w_{k}>0$ for large $k$ are negative (see [19]), that is, $0>w_{k}>\tilde{w}_{k}$. Then using the same argument as in the first part of the proof we have $0<\widetilde{v}_{k}<v_{k}$ for large $k$ for any solution $v$ of (5.15), that is, $\tilde{u}$ is the recessive solution of (5.14).

(iii) In [18], we posed the question whether the sequence $h_{k}:=k^{(p-1) / p}$ is the recessive solution of the difference equation

$$
\Delta\left(\Phi\left(\Delta x_{k}\right)\right)+c_{k} \Phi\left(x_{k+1}\right)=0, \quad c_{k}:=-\frac{\Delta\left(\Phi\left(\Delta h_{k}\right)\right)}{\Phi\left(h_{k+1}\right)}
$$

Now we can give the affirmative answer to this question for $p \geq 2$. It is shown in [18] that

$$
\begin{gathered}
u_{k}:=\Phi\left(\Delta h_{k}\right)=\left(\frac{p-1}{p}\right)^{p-1} k^{-(p-1) / p}\left[1+\frac{p-1}{2 p k}+o\left(k^{-1}\right)\right], \\
c_{k}=\frac{\gamma_{p}}{(k+1)^{p}}\left[1+O\left(k^{-1}\right)\right], \quad \gamma_{p}:=\left(\frac{p-1}{p}\right)^{p},
\end{gathered}
$$

both as $k \rightarrow \infty$. The sequence $u$ is a solution of the equation

$$
\Delta\left(c_{k}^{1-q} \Phi^{-1}\left(\Delta u_{k}\right)\right)+\Phi^{-1}\left(u_{k+1}\right)=0,
$$

which is reciprocal to (5.19) and $y_{k}=h_{k+1}=(k+1)^{(p-1) / p}$ is a solution of the equation

$$
\Delta\left(\Phi\left(\Delta y_{k}\right)\right)+c_{k+1} \Phi\left(y_{k+1}\right)=0
$$

which is reciprocal to (5.21) and differs from (5.19) only by the shift $k \rightarrow k+1$ in the sequence c. Since

$$
\sum^{\infty}\left(c_{k}^{1-q}\right)^{1-p}=\sum^{\infty} c_{k}<\infty
$$


assumption (5.12) is satisfied (with $q, c^{1-q}$, and 1 instead of $p, r$, and $c$, resp.), hence positive solutions of (5.21) are decreasing, that is, Theorems 4.1 and 4.2 apply to this case. By a direct computation, we have

$$
c_{k}^{1-q} u_{k} u_{k+1}\left|\Delta u_{k}\right|^{q-2} \sim k^{-p(1-q)} k^{-2(p-1) / p} k^{(-2 p+1)(q-2) / p}=k .
$$

This means, by Theorem 4.1, that if $q \in(1,2]$, then $u$ is the recessive solution of (5.21) and hence $y_{k}=h_{k+1}$ is the recessive solution of (5.22). Consequently, $h_{k}=k^{(p-1) / p}$ is the recessive solution of (5.19) if $p \geq 2$.

\section{Acknowledgments}

This research is supported by the Grant 201/07/0145 of the Czech Grant Agency of the Czech Republic, and the Research Project MSM0022162409 of the Czech Ministry of Education.

\section{References}

[1] P. Řehák, "Hartman-Wintner type lemma, oscillation, and conjugacy criteria for half-linear difference equations," Journal of Mathematical Analysis and Applications, vol. 252, no. 2, pp. 813-827, 2000.

[2] P. Řehák, "Oscillatory properties of second order half-linear difference equations," Czechoslovak Mathematical Journal, vol. 51, no. 2, pp. 303-321, 2001.

[3] P. Řehák, "Generalized discrete Riccati equation and oscillation of half-linear difference equations," Mathematical and Computer Modelling, vol. 34, no. 3-4, pp. 257-269, 2001.

[4] P. Řehák, "Oscillation criteria for second order half-linear difference equations," Journal of Difference Equations and Applications, vol. 7, no. 4, pp. 483-505, 2001.

[5] P. ̌̌ehák, "Oscillation and nonoscillation criteria for second order linear difference equations," Fasciculi Mathematici, no. 31, pp. 71-89, 2001.

[6] R. P. Agarwal, M. Bohner, S. R. Grace, and D. O'Regan, Discrete Oscillation Theory, Hindawi, New York, NY, USA, 2005.

[7] O. Došlý and P. Řehák, "Recessive solution of half-linear second order difference equations," Journal of Difference Equations and Applications, vol. 9, no. 1, pp. 49-61, 2003.

[8] M. Cecchi, Z. Došlá, and M. Marini, "Nonoscillatory half-linear difference equations and recessive solutions," Advances in Difference Equations, no. 2, pp. 193-204, 2005.

[9] M. Cecchi, Z. Došlá, and M. Marini, "Reciprocity principle and asymptotic properties of solutions of half-linear difference equations," submitted.

[10] M. Cecchi, Z. Došlá, M. Marini, and I. Vrkoč, "Asymptotic properties for half-linear difference equations," Mathematica Bohemica, vol. 131, no. 4, pp. 347-363, 2006.

[11] O. Došlý and Á. Elbert, "Integral characterization of the principal solution of half-linear second order differential equations," Studia Scientiarum Mathematicarum Hungarica, vol. 36, no. 3-4, pp. 455-469, 2000.

[12] M. Cecchi, Z. Došlá, and M. Marini, "Half-linear equations and characteristic properties of the principal solution," Journal of Differential Equations, vol. 208, no. 2, pp. 494-507, 2005.

[13] M. Cecchi, Z. Došlá, and M. Marini, "Corrigendum to: "Half-linear equations and characteristic properties of the principal solution"," Journal of Differential Equations, vol. 221, no. 1, pp. 272-274, 2006.

[14] M. Cecchi, Z. Došlá, and M. Marini, "Limit and integral properties of principal solutions for halflinear differential equations," Archivum Mathematicum, vol. 43, no. 1, pp. 75-86, 2007.

[15] Á. Elbert and T. Kusano, "Principal solutions of non-oscillatory half-linear differential equations," Advances in Mathematical Sciences and Applications, vol. 8, no. 2, pp. 745-759, 1998.

[16] O. Došlý and P. Řehák, Half-linear Differential Equations, vol. 202 of North-Holland Mathematics Studies, Elsevier, Amsterdam, The Netherlands, 2005.

[17] M. Cecchi, Z. Došlá, and M. Marini, “On recessive and dominant solutions for half-linear difference equations," Journal of Difference Equations and Applications, vol. 10, no. 9, pp. 797-808, 2004. 
[18] O. Došlý and S. Fišnarová, "Linearized Riccati technique and (non-)oscillation criteria for half-linear difference equations," Advances in Difference Equations, vol. 2008, Article ID 438130, 18 pages, 2008.

[19] W.-T. Li, "Classification schemes for nonoscillatory solutions of two-dimensional nonlinear difference systems," Computers \& Mathematics with Applications, vol. 42, no. 3-5, pp. 341-355, 2001. 